OPEN

SUBJECT AREAS:

SUPERCONDUCTING

PROPERTIES AND

MATERIALS

PHASE TRANSITIONS AND

CRITICAL PHENOMENA

Received

10 November 2014

Accepted

5 March 2015

Published

27 May 2015

Correspondence and requests for materials should be addressed to

E.S. (stavrou1@\|lnl.

gov)

\title{
Formation of As-As Interlayer Bonding in the collapsed tetragonal phase of $\mathrm{NaFe}_{2} \mathrm{As}_{2}$ under pressure
}

\author{
Elissaios Stavrou' , Xiao-Jia Chen ${ }^{2,1,3}$, Artem R. Oganov ${ }^{4,5,6}$, A. F. Wang 7 , Y. J. Yan 7 , X. G. Luo , X. H. Chen \\ \& Alexander F. Goncharov ${ }^{1,3,8}$
}

\begin{abstract}
${ }^{1}$ Geophysical Laboratory, Carnegie Institution of Washington, Washington, D.C. 20015, U.S.A, ${ }^{2}$ Center for High Pressure Science and Technology Advanced Research, Shanghai 201203, China, ${ }^{3}$ Key Laboratory of Materials Physics, Institute of Solid State Physics, CAS, Hefei, 230031, China, ${ }^{4}$ Department of Geosciences, Center for Materials by Design, Institute for Advanced Computational Science, Stony Brook University, Stony Brook, NY 11794-2100, U.S.A, ${ }^{5}$ Moscow Institute of Physics and Technology, 9 Institutskiy lane, Dolgoprudny city, Moscow Region, 141700, Russian Federation, 'School of Materials Science, Northwestern Polytechnical University, Xi'an, 710072, China, ${ }^{7}$ Hefei National Laboratory for Physical Science at Microscale and Department of Physics, University of Science and Technology of China, Hefei, Anhui 230026, China, ${ }^{8}$ University of Science and Technology of China, Hefei, 230026, China.
\end{abstract}

$\mathrm{NaFe}_{2} \mathrm{As}_{2}$ is investigated experimentally using powder $\mathrm{x}$-ray diffraction and Raman spectroscopy at pressures up to $23 \mathrm{GPa}$ at room temperature and using ab-initio calculations. The results reveal a pressure-induced structural modification at $4 \mathrm{GPa}$ from the starting tetragonal to a collapsed tetragonal phase. We determined the changes in interatomic distances under pressure that allowed us to connect the structural changes and superconductivity. The transition is related to the formation of interlayer As-As bonds at the expense of weakening of $\mathrm{Fe}$-As bonds in agreement with recent theoretical predictions.

$\mathrm{N}$ ature of superconductivity in iron-based superconductors (FeSCs) remain a challenge of modern condensed matter physics due to the complicated interplay between their structure, magnetism, electronic nematicity, and superconductivity ${ }^{1,2}$. A generic picture for the early discovered sister cuprate system is that superconductivity emerges and develops in a dome in the charge carrier concentration versus temperature phase diagram when magnetism of a parent compound is suppressed and a narrow spin glass state ${ }^{3}$ is passed. To study the emergence of superconductivity, one can naturally apply chemical doping, however application of pressure has the similar effect in FeSCs as there are some similarities for the structural distortions between pressure and chemical doping ${ }^{4}$. In the case of $122 \mathrm{FeSCs}$, pressure-induced suppression of antiferromagnetic spin fluctuations was proposed to account for the disappearance of superconductivity in a so-called collapsed tetragonal (CT) phase ${ }^{5}$. However, the structural modification has been suggested to be a key to the understanding of the superconductivity of iron pnictides ${ }^{6}$. A common structural characteristic of all Fe SCs is the presence of AsFe-As layers forming edge-sharing $\mathrm{FeAs}_{4}$ tetrahedra. It is believed that superconductivity emerges from these layers ${ }^{1,2}$.

$\mathrm{NaFe}_{2} \mathrm{As}_{2}$ crystallizes in the tetragonal (T) $\mathrm{ThCr}_{2} \mathrm{Si}_{2}$-type structure (I4/mmm) ${ }^{7,8}$ (see Fig. 1), belonging to the so-called 122 family of FeSCs. It has been characterized as the "missing" member of the 122 system, since initially it was thought that $\mathrm{Na}$, due to its small ionic radius, cannot fill the coordination sphere ${ }^{7,8}$ between the As-Fe-As layers. Moreover, $\mathrm{NaFe}_{2} \mathrm{As}_{2}$ has a remarkably high superconducting transition temperature $T_{c}$ of $25 \mathrm{~K}^{8}$ in comparison with less than $5 \mathrm{~K}$ in the case of $\mathrm{Cs}$ and $\mathrm{Rb}$ within this $\mathrm{AFe}_{2} \mathrm{As}_{2}$ family.

Previously reported structural studies on 122 FeSCs under pressure (e.g. Ref. 9) were limited to the cases of A being a divalent alkaline earth or rare earth metal atom, while systems with monovalent alkali metal, to the best of our knowledge, have not been studied. Although all previous studies suggested that the transformation from the $\mathrm{T}$ to the $\mathrm{CT}$ phase is a universal characteristic of divalent $\mathrm{AFe}_{2} \mathrm{As}_{2}$ compounds ${ }^{10}$, a detailed structural study on the atomic level and the link between structural modifications and superconductivity under pressure is still missing. In this paper we present a combined experimental, using $\mathrm{x}$-ray diffraction (XRD) and Raman spectroscopy, and theoretical study of $\mathrm{NaFe}_{2} \mathrm{As}_{2}$ under pressure. We examine the structural behavior of $\mathrm{AFe}_{2} \mathrm{As}_{2}$ superconductors under pressure in the case of $\mathrm{A}$ being an alkali metal to obtain a deeper insight on the interplay between structure and superconductivity under pressure. We present, for the first time, direct experimental evidence of the 


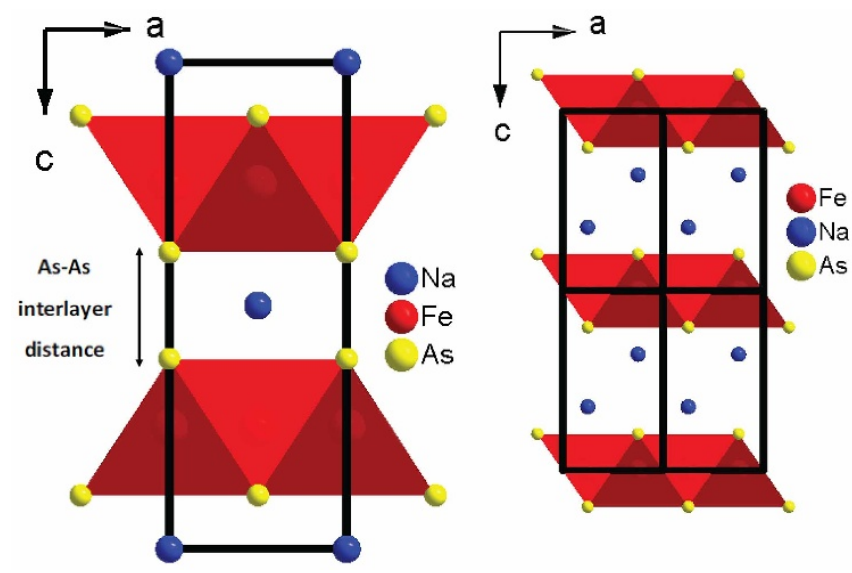

Figure $1 \mid$ (a) $\mathrm{ThCr}_{2} \mathrm{Si}_{2}$-type crystal structure of $\mathrm{NaFe}_{2} \mathrm{As}_{2}$, (b) $\mathrm{PbClF}-$ type crystal structure of NaFeAs.

formation of As-As interlayer bonds under pressure. According to theoretical predictions ${ }^{11,12}$ this is the key parameter determining the correlation between structure and superconductivity for 122 SC.

\section{Results}

$\mathrm{X}$-ray diffraction results and analysis. Figure 2 shows selected XRD patterns of $\mathrm{NaFe}_{2} \mathrm{As}_{2}$ obtained at various pressures up to $23 \mathrm{GPa}$. All the observed peaks in this pressure range can be properly indexed according to the tetragonal $\mathrm{ThCr}_{2} \mathrm{Si}_{2}$-type structure. On the other hand, an unusual pressure behavior of the Bragg peaks related with the $a$-axis can be clearly seen at low pressures. This is highlighted by the merge of the (114) and (200) peaks at $2 \theta \approx 10^{\circ}$ and the splitting of the peak at $2 \theta \approx 7^{\circ}$ into its components, (110) and (103). We observe a decrease of the $2 \theta$ position of the (200) peak and a practically constant position of the (101) peak, while the $2 \theta$ positions of all other peaks [e.g. (002)] shift towards higher values.

In order to determine the positional parameters and the interatomic distances we have performed a detailed Rietveld refinement of the XRD patterns using the GSAS program ${ }^{13}$. Typical refined profiles are shown in Fig. 3 at 1.0 and $5.1 \mathrm{GPa}$. The only free positional parameter of this structure is the $z$ position of As atoms, Wyckoff position (WP) $4 e(0,0, z)$, since positions of $\mathrm{Na}$ and $\mathrm{Fe}$ atoms are

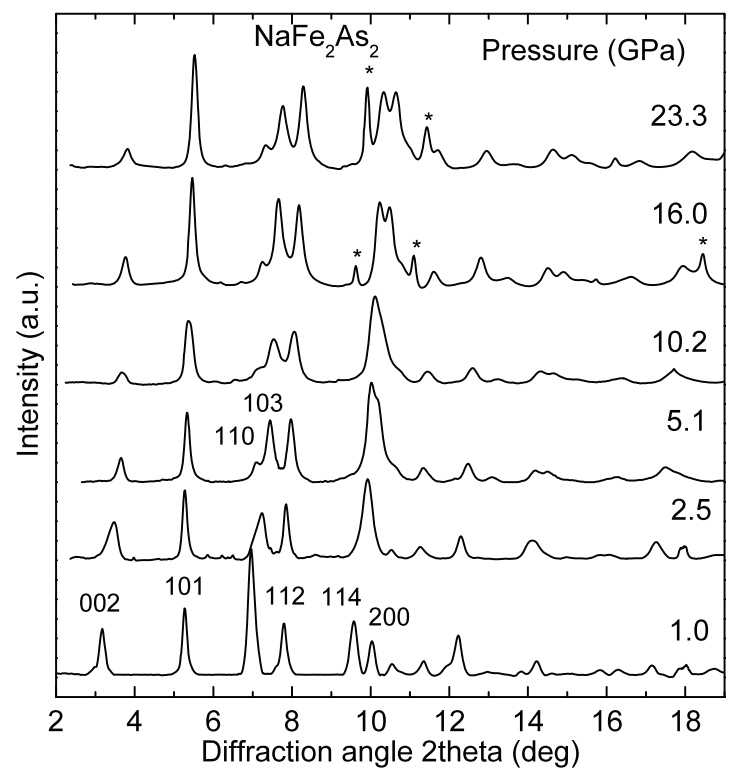

Figure $2 \mid \mathrm{X}$-ray diffraction patterns of $\mathrm{NaFe}_{2} \mathrm{As}_{2}$ at various pressures. The asterisks denote peaks from $\mathrm{Ne}$.
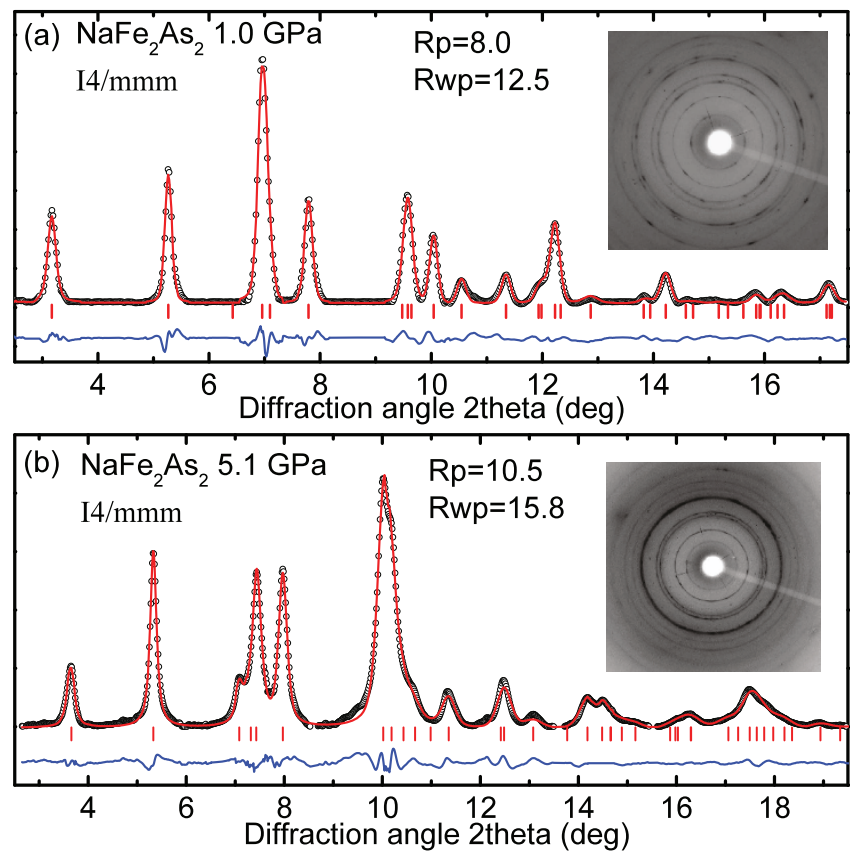

Figure $3 \mid$ Rietveld refinements for $\mathrm{NaFe}_{2} \mathrm{As}_{2}$ at (a) $1.0 \mathrm{GPa}$ and (b) 5.1 GPa. Respective 2D x-ray diffraction images are shown in insets.

fixed. Our two-dimensional (2D) images reveal an almost perfect ring shape of reflections, mainly due to the very fine powder used in this study. In addition, wide angle dispersive XRD measurements helped us to overcome any intensity redistribution caused by preferred orientation. Moreover, XRD patterns have been collected from several spots of the sample at the same pressure, and the results were averaged. The agreement between experimental and theoretical interatomic distances provides an additional confidence of these results.

From XRD data of $\mathrm{NaFe}_{2} \mathrm{As}_{2}$ we have obtained the lattice parameters and the unit cell volume as a function of pressure, and the results are shown in the plots of Fig. 4 . The pressure evolution of the lattice parameters and the $c / a$ ratio changes at a critical pressure $P_{c} \approx$ $4 \mathrm{GPa}$. Below $P_{c}, a$-axis increases while both the $c$-axis and the $c / a$ ratio decrease rapidly. In contrast above $P_{c}, a$-axis starts to decrease and $c$-axis becomes much less compressible. Consequently the $c / a$ ratio shows a gradual decrease. The different behavior of $c$-axis, below and above $P_{c}$, can be viewed as a modification of the initial tetragonal phase (T) to the so-called collapsed tetragonal (CT) phase (e.g. Ref. 14). We have fitted the pressure-volume data by the thirdorder Birch equation of state ${ }^{15}$ and determined the bulk modulus $B_{0}$ and its first pressure derivative $B_{0}^{\prime}$ (Fig. 4). The CT phase is much less compressible compared to the T phase as $B_{0}$ increases by a factor of 5 . No apparent volume discontinuity is observed at this isostructural phase transition.

The results from the theoretical predictions are also plotted in Fig. 4 for both a magnetic (M) and a non-magnetic phase (NM). In the case of the $M$ phase, a ferromagnetic configuration has been used and the energy difference from the antiferromagnetic configuration is very small. It is well known that density-functional calculations have a systematic error of several GPa in the equation of state, and a simple constant shift usually brings computed equations of state into close agreement with experiment ${ }^{16}$. Here, the theoretical pressures were corrected by $4 \mathrm{GPa}$. With this correction, an almost perfect agreement between experimental and theoretical values (for both phases) can be clearly seen from Fig. 4 . It is noteworthy that the $\mathrm{M}$ phase, although it has higher volume at low pressures, is more stable than the NM one, due to the magnetic interactions which result in a (slightly) lower enthalpy up to well above $P_{c}$. Moreover, 

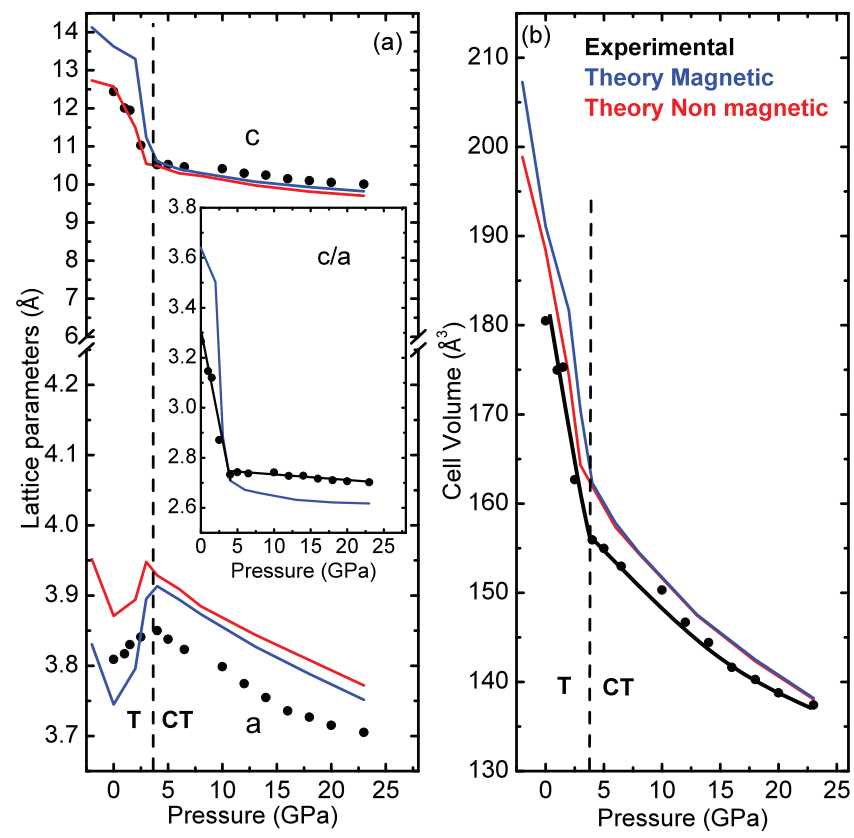

Figure $4 \mid$ Pressure dependence of (a) lattice parameters and (b) cell volume of $\mathrm{NaFe}_{2} \mathrm{As}_{2}$. The inset shows the pressure dependence of the $c / a$ ratio. Experimental data are shown with solid symbols and theoretical predictions ( shifted by 4 GPa to higher P, see text) with blue (M-phase) and red (NM-phase) lines, respectively. The solid black curves in (b) are the third-order Birch-Murnagan EOS fits for the T $\left(B_{0}=22 \mathrm{GPa}, B=4\right)$ and $\mathrm{CT}\left(B_{0}=110 \mathrm{GPa}, B=4.5\right)$ phases.

both phases exhibit a T to CT transformation at the same pressure. In the magnetic case (which corresponds to observations of Ref. 7) our calculations suggest that $\mathrm{CT}$ phase remains ferromagnetic at $\mathrm{T}=$ $0 \mathrm{~K}$. Since theory predicts the same phase transformation in magnetic and nonmagnetic cases, we can conclude that the structural behavior is not driven by magnetic interactions.

Raman spectroscopy. Raman spectra of $\mathrm{NaFe}_{2} \mathrm{As}_{2}$ under pressure show three out of four Raman-active zone-center modes predicted from group theory ${ }^{17}: A_{1 g}(\mathrm{As}(\mathrm{z})), B_{1 g}(\mathrm{Fe}(\mathrm{z}))$ and $E_{g}(\mathrm{As}-\mathrm{Fe}(\mathrm{xy})$ and FeAs(xy)) (Fig. 5(a)). $A_{1 g}$ and $B_{1 g}$ modes correspond to the displacement of $\mathrm{As}$ and $\mathrm{Fe}$ atoms along the $c$-axis, respectively. The two $E_{g}$ modes correspond to mixed lateral $\mathrm{As}$ and $\mathrm{Fe}$
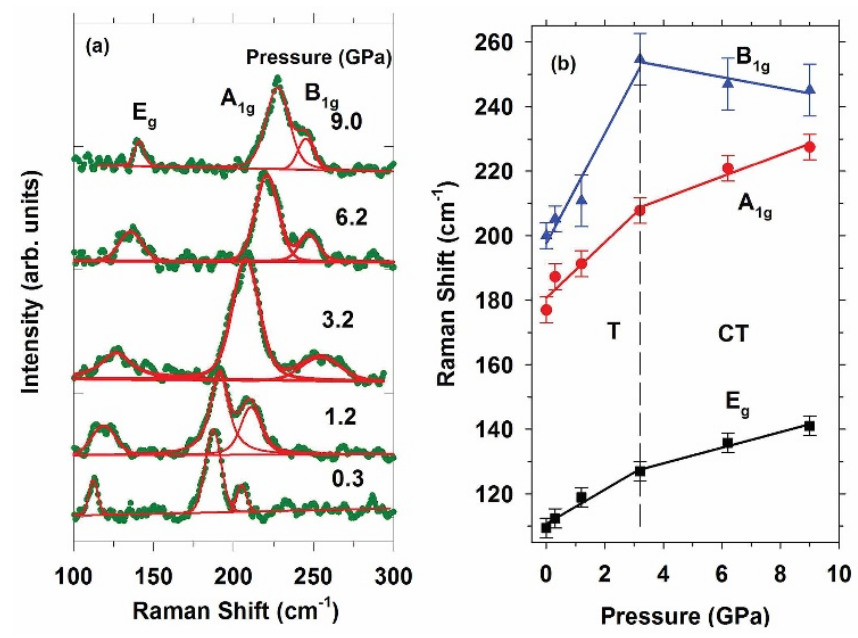

Figure $5 \mid$ (a) Raman spectra of $\mathrm{NaFe}_{2} \mathrm{As}_{2}$ at various pressures. (b) Raman peaks frequencies plotted against pressure. In (b) lines are the results of linear fits. displacements inside the As and Fe layers. The ambient pressure frequencies are in very good agreement with previous studies ${ }^{17}$ with the exception of the very low intensity high frequency $E_{g}(\mathrm{Fe}-$ As(xy)) mode, which was not observed. All Raman modes show normal mode behavior i.e. increase of the frequency with increasing pressure in phase $\mathrm{T}$ (Fig. 5(b)). At $3 \mathrm{GPa}$, a critical pressure which is in agreement with that determined from XRD measurements, there is an apparent change in the pressure slope of Raman modes (see Table 1), accompanied by an increase of the linewidth. Remarkably, the $B_{1 g}(\mathrm{Fe})$ mode shows a softening in CT phase. The mode Grüneisen parameters $\left(\gamma_{T}\right)$ determined using the experimental results of this work are shown Table 1. The $\gamma_{T}$ parameters of modes of the $\mathrm{T}$ phase are common for materials with mixed covalent-ionic bonding (such as within the As-Fe-As layers), due to the presence of weaker interlayer bonds, which experience a larger compression. In the CT phase, the $A_{1 g}$ (As) mode exhibit a higher $\gamma_{T}$ which almost doubles. These changes suggests an alteration of this mode character from intralayer to interlayer, which results in an increase of the contraction of the respective bond. Similarly, we find the doubling of the $\gamma_{T}$ of the intralayer $E_{g}$ (As-Fe) mode. It is the most intriguing that the $\gamma_{T}$ of the $B_{1 g}$ (Fe) mode becomes negative, in CT phase suggesting weakening of bonds under pressure.

\section{Discussion}

Our x-ray diffraction results reveal the behavior of the As-As interlayer distance in comparison with the $\mathrm{Fe}$-As intratetrahedral distance (see Fig. 6). Figure 7 shows the experimentally and theoretically determined values of these distances under pressure. An almost perfect agreement between the two sets of values is observed. The As-As interlayer distance decreases rapidly with pressure up to $4 \mathrm{GPa}$. This decrease is almost half of that of c-axis and it accounts for the whole c-axis contraction while the thickness of As-layers (vertical distance between As-As layers of the same As-Fe-As block) remains almost constant or even slightly increases. Shortening of c-axis is driven exclusively by the distance between the tetrahedra blocks $\left(c=2 d_{A s-A s(c)}+2 d_{A s-F e-A s(c)}\right)$. The interlayer As-As and the intralayer Fe-As distances become practically equal above $4 \mathrm{GPa}$, i.e. after the transformation to the $\mathrm{CT}$ phase. The As-As distance above $4 \mathrm{GPa}$ takes a value (2.4 $\AA$ in experiment and $2.6 \AA$ in theory) which is consistent with the formation of As-As bond (covalent radius of As is $1.2 \AA^{18}$ ). Thus, we conclude that a major change in bonding between As atoms occurs under pressure. It can be viewed as a change from 4 -fold to 5 -fold coordination with formation of interlayer As-As bonds (see Fig. 6(b)). This conclusion is further justified by the doubling of the $\gamma_{T}$ of the $A_{1 g}$ mode after the phase transition. In contrast the decrease of the $\gamma_{T}$ of the $B_{1 g}$ mode indicates weakening of the respective Fe bond.

Previous experimental studies on $\mathrm{AFe}_{2} \mathrm{As}_{2}\left(\mathrm{~A}=\mathrm{Eu}^{10}, \mathrm{Ba}^{19}, \mathrm{Sr}^{20}\right.$, $\left.\mathrm{Ca}^{14}\right)$ compounds, reveal a common structural trend under pressure. This is an increase of $a$ while both the $c$ and $c / a$ ratio decrease rapidly below $P_{c}$, and a decrease of $a$ and much less compressible $c$ above $P_{c}$. In the light of this observation, Uhoya et al. ${ }^{10}$ suggested that the transformation from the $\mathrm{T}$ to the $\mathrm{CT}$ phase is a universal characteristic of $\mathrm{AFe}_{2} \mathrm{As}_{2}$ compounds, while the value of $P_{c}$ depends on the divalent metal. Our study reveal a similar behavior for single valence metal suggesting that this universality is also independent of the valence of A metal. Transformation to the CT phase with further increasing pressure always suppresse ${ }^{19}$ or diminishes superconductivity $^{20}$. In a study of $\mathrm{CaFe}_{2} \mathrm{As}_{2}$ under pressure ${ }^{14}$ it has been suggested that one of the main structural differences between $\mathrm{T}$ and CT phases is the value of the As-Fe-As tetrahedral angles. Both tetrahedral angles change abruptly during the phase transition from a value very close (T) to a value far away (CT) from the ideal tetrahedral angle $\left(109.5^{\circ}\right)$. Since $T_{c}$ decreases with increasing deviation from the ideal tetrahedral angles, it has been concluded that this is crucial for 
Table $1 \mid$ Assignments, ambient conditions frequencies $\omega_{0}$ (in $\left.\mathrm{cm}^{-1}\right)$, slopes $(\partial \omega / \partial P)_{T}\left(\right.$ in $\left.\mathrm{cm}^{-1} \mathrm{GPa}^{-1}\right)$ and Grüneisen parameters $\gamma_{T}=-\partial(\ln \omega) /\left.\partial(\ln V)\right|_{T}$ of the Raman modes of the T and CT phases

$\mathrm{T}$

\begin{tabular}{lccc}
\hline Mode & $\omega_{0}$ & $(\partial \omega / \partial P)_{T}$ & $\gamma_{T}$ \\
\hline$E_{g}$ & 110.7 & 5.3 & 1.05 \\
$A_{l g}$ & 180.7 & 8.6 & 1.05 \\
$B_{1 g}$ & 200.9 & 17.0 & 1.86 \\
\hline
\end{tabular}

CT

\begin{tabular}{ccc}
\hline$\omega_{0}$ & $(\partial \omega / \partial P)_{T}$ & $\gamma_{T}$ \\
119.8 & 2.4 & 2.2 \\
197.8 & 3.4 & 1.9 \\
259.2 & -1.7 & -0.72
\end{tabular}

superconductivity although the direct link and the underling mechanism which correlates tetrahedral angles and superconductivity is not clear.

Recent theoretical studies ${ }^{21,11,12}$ were focused on the change in interatomic distances under pressure, mainly on the interlayer AsAs distance (noted as As-As (c) in Fig. 6). This distance has been predicted to decrease abruptly with pressure, reaching a value that is very close to the As-As covalent bond distance in the CT phase. Consequently it has been proposed that the transition to the CT phase is induced by formation of a direct As-As interlayer bond and a weakening of the in-plane $\mathrm{Fe}-\mathrm{Fe}^{11}$ and $\mathrm{Fe}-\mathrm{As}$ bonds ${ }^{12}$. It is noteworthy that in the case of 111 superconductors (e.g. Ref. 22) the Fe-As-Fe layers are separated by two layers of cations (see Fig. 1). Thus, an interaction between As atoms of different layers is hindered and only intralayer modifications can affect superconductivity.

The spin-state of $\mathrm{Fe}$ is the key parameter which controls As-As bonding and consequently the lattice parameters ${ }^{12}$. Under compression the magnetic moment of Fe decreases and consequently, the strength of the Fe-As bonding (see Fig. 4 of Ref. 12). This is also evident from our calculations, see Fig. 8. The results of our calculations suggest that the CT phase is magnetic i.e. the Fe magnetic moment is not vanished but suppressed after the transformation decreasing the strength of the Fe-As bonding in agreement with softening of the $B_{1 g}$ Raman mode (Fig. 5). The structural changes determined in this paper (Fig. 7) clearly support this scenario. A direct consequence of the As 5-fold coordination is that the system loses its two-dimensionality (Fig. 6(b)), and with it, superconductivity. Moreover, the weakening of the in-plane Fe-Fe bonds ${ }^{11}$ in the CT phase also decreases superconductivity.

From our data we find that the tetrahedral angles change continuously with pressure (inset to Fig. 7) in a manner very similar to that determined previously in $\mathrm{CaFe}_{2} \mathrm{As}_{2}{ }^{14,9}$ and also predicted by theory ${ }^{12}$. During the initial compression the a-axis expands and the $c$-axis rapidly decreases due to the development of the As-As interlayer bonding and this keeps $\mathrm{Fe}$-As distances almost constant. In addition,
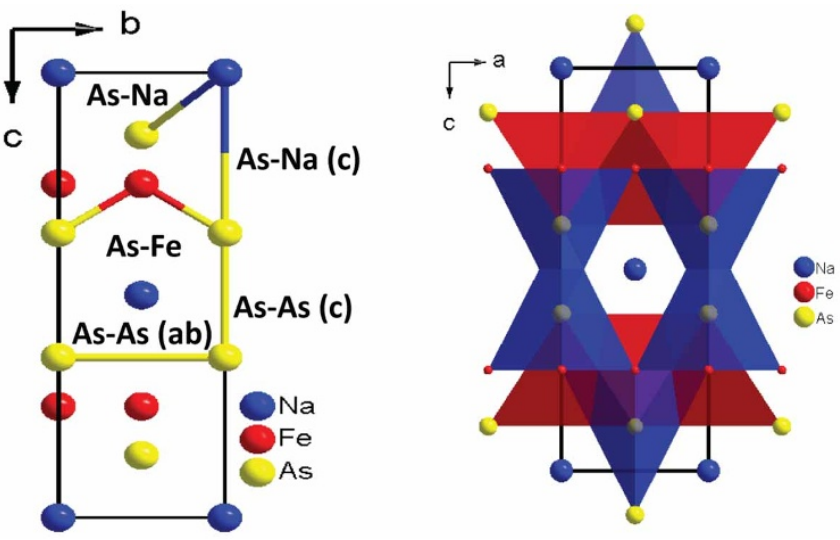

Figure 6 | (a) $\mathrm{ThCr}_{2} \mathrm{Si}_{2}$-type crystal structure of $\mathrm{NaFe}_{2} \mathrm{As}_{2}$ showing the different interatomic distances and (b) $\mathrm{ThCr}_{2} \mathrm{Si}_{2}$-type crystal structure of $\mathrm{NaFe}_{2} \mathrm{As}_{2}$ with As atoms in 5 -fold coordination. the tetrahedral angles are close to, or even approach, the ideal value. Above $\mathrm{P}_{c}$, the tetrahedral angles take value away from the ideal because $a$-axis stops to increase to mediate the $c$-axis decrease. So,

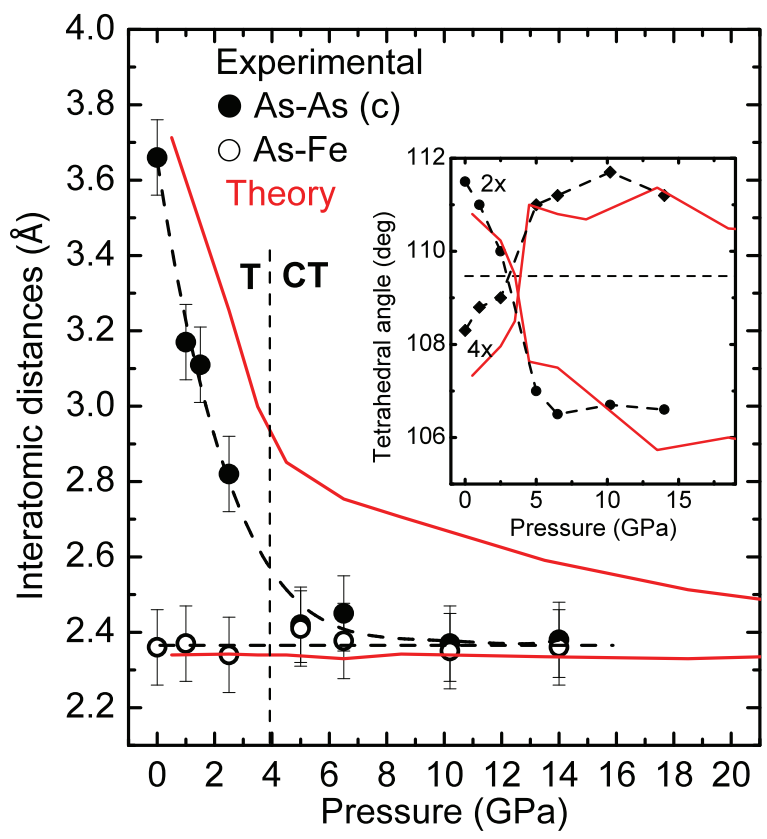

Figure $7 \mid$ Pressure dependence of selected interatomic distances of $\mathrm{NaFe}_{2} \mathrm{As}_{2}$ : As-As interlayer along the $c$ axis and Fe-As inside the FeAs 4 tetrahedra. Experimental and theoretical values are shown with black symbols and red lines respectively, the dashed lines are guides for the eye. The inset shows the pressure dependence of the two distinct tetrahedral AsFe-As bond angles. The ideal tetrahedral angle is indicated by the horizontal dashed line.

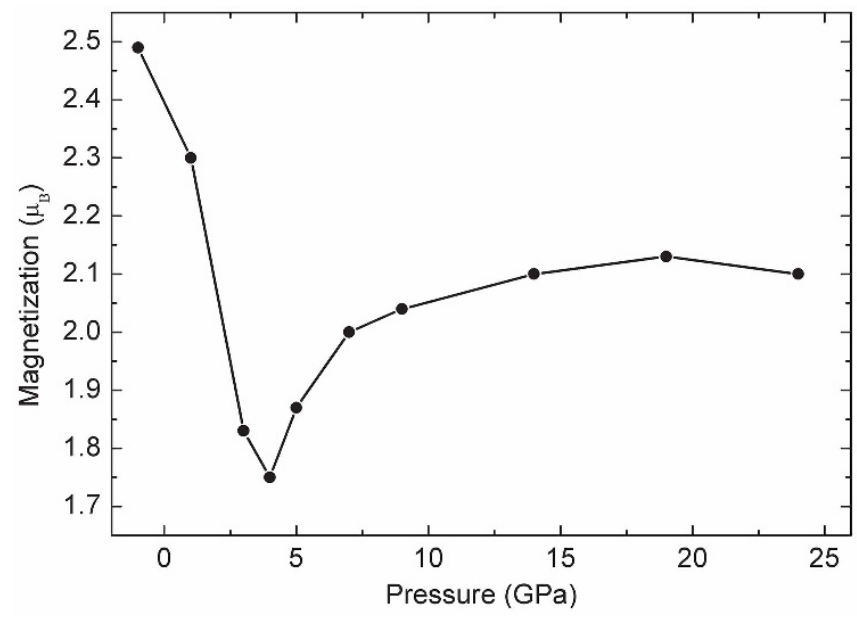

Figure 8 Calculated magnetization per unit cell of $\mathrm{NaFe}_{2} \mathrm{As}_{2}$ as a function of pressure. 
the correlation between the deviation of tetrahedral angles and $T_{c}$ turns out to be a "side effect" of the change of As atom position with increasing pressure which primary affect $T_{c}$.

In conclusion, our study reveals, for the first time, the strong experimental evidence supporting the theoretically suggested mechanism controlling the structural behavior of $\mathrm{AFe}_{2} \mathrm{As}_{2}$ compounds under pressure. Moreover the concomitant experimental and theoretical study of $\mathrm{NaFe}_{2} \mathrm{As}_{2}$ provides, missing up to now, a direct link between structural characteristics and superconductivity under pressure.

\section{Methods}

Experimental study. Fine powder prepared of $\mathrm{NaFe}_{2} \mathrm{As}_{2}$ single crystals were loaded into a diamond anvil cell (DAC) for the angle dispersive XRD experiments. For the high-pressure Raman measurements, small pieces with a typical dimension of $50 \mu \mathrm{m}$ were loaded into a DAC. Neon was used as the pressure-transmitting medium for both sets of measurements. Small quantities of ruby and gold powder were also loaded, for determination of pressure through ruby luminescence and gold equation of state (EOS), respectively. XRD data were collected at the GSECARS (sector 13), using a MAR355 CCD detector. The monochromatic $\mathrm{x}$-ray beam $(\lambda=0.3344 \AA$ ) was focused to a nominal diameter of $4 \mu \mathrm{m}$. The images were integrated using the FIT2 ${ }^{23}$ program to yield intensity versus $2 \theta$ diagrams. Raman spectra were measured in a 135 degree $^{24}$ geometry using the $532 \mathrm{~nm}$ line from a solid state laser for excitation. Ultralow fluorescence type IIa synthetic diamond anvils were used for Raman experiments.

Single crystals in the Na-Fe-As system were grown by use of the NaAs flux method. We obtained NaAs by reacting the mixture of the elemental $\mathrm{Na}$ and As in an evacuated quartz tube at $200^{\circ} \mathrm{C}$ for $10 \mathrm{~h}$. NaAs and Fe powders were carefully weighed and thoroughly ground. The mixtures were put into alumina crucibles and then sealed in iron crucibles under $1.5 \mathrm{~atm}$ of highly pure argon gas. The crucibles were sealed and heated to $950^{\circ} \mathrm{C}$ at a rate of $60^{\circ} \mathrm{C} / \mathrm{h}$ in the tube furnace filled with the inert atmosphere and kept at $950^{\circ} \mathrm{C}$ for $10 \mathrm{~h}$ and then cooled slowly to $600^{\circ} \mathrm{C}$ at $3^{\circ} \mathrm{C} / \mathrm{h}$ to grow single crystals. The shiny crystals with typical size of $6 \times 6 \times 0.2 \mathrm{~mm}$ can be easily cleaved from the melt.

Theoretical calculations. Our theoretical calculations were performed using density functional theory within the generalized gradient approximation ${ }^{25}$ and using the PAW method ${ }^{26}$ as implemented in the VASP code ${ }^{27}$. The PAW potentials used had [Be] core (outermost radius 2.2 a.u.) for $\mathrm{Na}$ atoms, [ $\mathrm{Ar}$ ] core (radius 2.3 a.u.) for $\mathrm{Fe}$ atoms, and [Ni] core (radius 2.1 a.u.) for As atoms. We used the plane wave kinetic energy cutoff of $360 \mathrm{eV}$ and $6 \times 6 \times 3 \Gamma$-centered meshes for Brillouin zone sampling. Electronic optimization was done self-consistently with a threshold of $10^{-4} \mathrm{eV} / \mathrm{cell}$, and structure relaxation proceeded until changes in the enthalpy were below $10^{-3} \mathrm{eV} /$ cell. A ferromagnetic configuration with all $\mathrm{Fe}$ atoms given a starting magnetic moment of 2 Bohr magnetons was used. The starting magnetic moment plays little role (only the magnetic symmetry is important) and was optimized during electronic and structural relaxation.

1. Mazin, I. I. Superconductivity gets an iron boost. Nature 464, 183 (2010).

2. Paglione, J. \& Greene, R. L. High-temperature superconductivity in iron-based materials. Nat. Phys. 6, 645 (2010).

3. Dioguardi, A. P. et al. Coexistence of cluster spin glass and superconductivity in $\mathrm{Ba}$ $\left(\mathrm{Fe}_{1-x} \mathrm{Co}_{x}\right)_{2} \mathrm{As}_{2}$ for $0.060 \leq \mathrm{x} \leq 0.071$. Phys. Rev. Lett. 111, 207201 (2013).

4. Kimber et al., S. A. J. Similarities between structural distortions under pressure and chemical doping in superconducting $\mathrm{BaFe}_{2} \mathrm{As}_{2}$. Nat. Mater. 8, 471 (2009).

5. Pratt, D. K. et al. Suppression of antiferromagnetic spin fluctuations in the collapsed phase of $\mathrm{CaFe}_{2} \mathrm{As}_{2}$. Phys. Rev. B 79, 060510 (2009).

6. Stewart, G. R. Superconductivity in iron compounds. Rev. Mod. Ph. 83, 1589 (2011).

7. Friederichs, G. M. et al. Metastable $11 \mathrm{k}$ superconductor $\mathrm{Na}_{1 y} \mathrm{Fe}_{2 x} \mathrm{As}_{2}$. Inor. Chem. 51, 8161-8167 (2012).

8. Gooch, M. et al. Superconductivity in ternary iron pnictides: $\mathrm{AFe}_{2} \mathrm{As}_{2}(\mathrm{~A}=$ alkali metal) and LiFeAs. Physica C 470, S276 (2010).

9. Mittal, R. et al. Ambient- and low-temperature synchrotron $\mathrm{x}$-ray diffraction study of $\mathrm{BaFe}_{2} \mathrm{As}_{2}$ and $\mathrm{CaFe}_{2} \mathrm{As}_{2}$ at high pressures up to $56 \mathrm{GPa}$. Phys. Rev. B 83, 054503 (2011).

10. Uhoya, W. et al. Anomalous compressibility effects and superconductivity of $\mathrm{EuFe}_{2} \mathrm{As}_{2}$ under high pressures. J. Phys.: Condens. Matter 22, 292202 (2010).

11. Colonna, N., Profeta, G., Continenza, A. \& Massidda, S. Structural and magnetic properties of $\mathrm{CaFe}_{2} \mathrm{As}_{2}$ and $\mathrm{BaFe}_{2} \mathrm{As}_{2}$ from first-principles density functional theory. Phys. Rev. B 83, 094529 (2011).
12. Yildirim, T. Strong coupling of the Fe-spin state and the As-As hybridization in iron-pnictide superconductors from first-principle calculations. Phys. Rev. Lett. 102, 037003 (2009)

13. Larson, A. C. \& Von Dreele, R. B., General structure analysis system (GSAS). Los Alamos National Laboratory Report LAUR 86-748 (1994). Available at: http:// www.ccp14.ac.uk/solution/gsas/ (Accessed: 4th February 2015).

14. Kreyssig, A. et al. Pressure-induced volume-collapsed tetragonal phase of $\mathrm{CaFe}_{2} \mathrm{As}_{2}$ as seen via neutron scattering. Phys. Rev. B 78, 184517 (2008).

15. Birch, F. Finite strain isotherm and velocities for single-crystal and polycrystalline $\mathrm{NaCl}$ at high-pressures and 300K. J. Geophys. Res. 83, 1257 (1978).

16. Oganov, A., Brodholt, J. \& Price, G. Ab initio elasticity and thermal equation of state of $\mathrm{MgSiO}_{3}$ perovskite. Earth Planet. Sci. Lett. 184, 555-560 (2001).

17. Litvinchuk, A. P. et al. Raman-scattering study of $\mathrm{K}_{x} \mathrm{Sr}_{1-x} \mathrm{Fe}_{2} \mathrm{As}_{2}(\mathrm{x}=0.0,0.4)$ Phys. Rev. B 78, 060503 (2008).

18. Cordero, B. et al. Covalent radii revisited. Dalton Trans. 21, 2832-2838 (2008).

19. Uhoya, W. et al. Collapsed tetragonal phase and superconductivity of $\mathrm{BaFe}_{2} \mathrm{As}_{2}$ under high pressure. Phys. Rev. B 82, 144118 (2010).

20. Uhoya, W. O. et al. Phase transition and superconductivity of $\mathrm{SrFe}_{2} \mathrm{As}_{2}$ under high pressure. J. Ph. Condens. Matter. 23, 122201 (2011).

21. Sanna, A., Profeta, G., Massidda, S. \& Gross, E. K. U. First-principles study of rareearth-doped superconducting $\mathrm{CaFe}_{2} \mathrm{As}_{2}$. Phys. Rev. B 86, 014507 (2012).

22. Zhou, X. et al. Evolution from unconventional spin density wave to superconductivity and a pseudogaplike phase in $\mathrm{NaFe}_{1-x} \mathrm{Co}_{x} \mathrm{As}$. Phys. Rev. Lett. 109, 037002 (2012)

23. Hammersley, A. P., Svensson, S. O., Hanfland, M., Fitch, A. N. \& Hausermann, D. High Pres. Res. 14, 235 (1996).

24. Goncharov, A. F. \& Struzhkin, V. V. Raman spectroscopy of metals, hightemperature superconductors and related materials under high pressure. J. Raman Spectrosc. 34, 532-548 (2003).

25. Perdew, J. P., Burke, K. \& Ernzerhof, M. Generalized gradient approximation made simple. Phys. Rev. Lett. 77, 3865 (1996).

26. Kresse, G. \& Joubert, D. From ultrasoft pseudopotentials to the projector augmented-wave method. Phys. Rev. B 59, 1758 (1999).

27. Kresse, G. \& Furthmuller, J. Efficient iterative schemes for ab initio total-energy calculations using a plane-wave basis set. Phys. Rev. B 54, 11169-11186 (1996).

\section{Acknowledgments}

This work was supported by the DARPA (Grants No. W31P4Q1310005 and No. W31P4Q1210008), Carnegie Canada, EFree- the DOE EFRC center at Carnegie, the Government of the Russian Federation (No. 14.A12.31.0003) and the Ministry of Education and Science of Russian Federation (Project No. 8512). GSECARS is supported by the U.S. NSF (EAR-0622171, DMR-1231586) and DOE Geosciences (DE-FG02-94ER14466). Use of the APS was supported by the DOE-BES under Contract No. DE-AC02-06CH11357. Calculations were performed on XSEDE facilities and on the cluster of the Center for Functional Nanomaterials, BNL, which is supported by the DOE-BES under contract no. DE-AC02-98CH10086. Sample growth was supported by the Natural Science Foundation of China, the "Strategic Priority Research Program (B)" of the Chinese Academy of Sciences, and the National Basic Research Program of China.

\section{Author contributions}

E.S., X.-J.C and A.G designed the research. E.S. and A.G. conducted the experiments and analyzed the experimental data. A.R.O. performed the calculations. A.F.W., Y.J.Y., X.G.L. and X.H.C. prepared the single crystals $\mathrm{NaFe}_{2} \mathrm{As}_{2}$. E.S., X.-J.C, A.G and A.R.O. contributed in writing the manuscript, all authors reviewed the manuscript.

\section{Additional information}

Competing financial interests:The authors declare no competing financial interests.

How to cite this article: Stavrou, E. et al. Formation of As-As Interlayer Bonding in the collapsed tetragonal phase of $\mathrm{NaFe}_{2} \mathrm{As}_{2}$ under pressure. Sci. Rep. 5, 9868; DOI:10.1038/ srep09868 (2015).

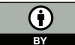

This work is licensed under a Creative Commons Attribution 4.0 International License. The images or other third party material in this article are included in the article's Creative Commons license, unless indicated otherwise in the credit line; if the material is not included under the Creative Commons license, users will need to obtain permission from the license holder in order to reproduce the material. To view a copy of this license, visit http://creativecommons.org/licenses/by/4.0/ 